\title{
Research on Development of Non-profit Organizations in Suzhou of China during the Transitional Period
}

\author{
Xueying Tian* \\ School of Business, Suzhou University of Science and Technology, 1 Kerui Rd. \\ Suzhou, Jiangsu, China 215009 \\ lucy.txy@163.com
}

\begin{abstract}
The economy in Suzhou, China is in the late stages of its industrialisation, during which, the completeness of non-profit organizations (NPOs) has been an important indicator for measuring the all-round socio-economic development of Suzhou. Currently, the NPOs of Suzhou make up for deficiencies in the government and the market, and thus make a positive contribution to the political and economic development of Suzhou. However, it is easy to find that NPOs still suffer disadvantages such as slow development, rarity, and low capacity: these inhibit their active benefits. This research investigated the classification and development status of the NPOs in Suzhou; on this basis, it analyzed the main problems facing them. Moreover, aiming at these problems, countermeasures and suggestions were proposed so as to allow them to play a more important in the socioeconomic system of Suzhou.
\end{abstract}

Keywords: Non-profit organizations, economic development, industrialisation

\section{Introduction}

With the societal progress, non-profit organizations (NPOs), as a new-type of independent organization, play an increasingly important role in the socio-economic system. In recent years, the economy of Suzhou has been in the late stages of industrialisation, during which, the coordinated development of both economy and society has been a focus drawing high attention from many quarters. Furthermore, instead of GDP which is an important indicator for measuring overall socio-economic development, the completeness of an NPO has become a good indicator of social modernisation. Currently, NPOs in Suzhou have developed vigorously and involve various fields including politics, economy, education, science and technology, culture, sports, health, social welfare, etc. In addition to playing their own roles, these NPOs make a positive contribution to the political and economic development of Suzhou by making up for deficiencies in the government and the market. It can be said that the development of NPOs is expected to exert a significant effect on the coordinated development of the socio-economic status of Suzhou in the future. However, the NPOs of Suzhou still present obvious shortcomings such as slow development, small per capita numbers, low selfdevelopment capacity, and weak oversight. These problems restrict their realisation of their intended roles, and thereby extremely limit their influence and degree of identification. Therefore, clarifying the role and position of the NPOs in the socioeconomic system of Suzhou and analysing their development strategies are worthy of research as a prompt solution to these problems would help the modernisation of Suzhou.

According to the functional orientation and classification of NPOs in Suzhou, this research analyzed their development status and the problems facing them. Based on the analysis, corresponding suggestions were proposed to maximise the important role played by NPO in the socio-economic system of Suzhou. 


\section{Classification and Development Status of NPOs in Suzhou}

\subsection{Classification}

In the exploration of the functions and classifications of NPOs, Wang (2007) listed four functions of NPOs in the management of public affairs: 1) a service function, by taking parts of government functions and directly serving various social public affairs; 2) an intermediary function, linking government with enterprises, government with society, and China with other countries; 3 ) supervising the government and market by acting as a third party; 4) stability and security functions for guaranteeing the basic living standards of citizens and supporting vulnerable groups. In accordance with these aforementioned functions, NPOs are divided into the following types: coordinated social groups, private non-enterprises providing direct services, foundations providing capital support, and institutions. Wang Ming (2006) held that four social functions of NPOs, including mobilising social resources, providing social services, coordinating and managing society, and advocating and influencing policies could be summed up in terms of input, output, influence, effect, etc.

Based on the aforementioned classifications and the socio-economic development in Suzhou, the author divided NPOs into three categories according to their social functions: (1) public welfare, providing financial aid; (2) providing social services; and (3) those coordinating and managing aspects of society.

The public services provided by NPOs involve, and are mainly concentrated on, the following: public charity, disaster relief, poverty alleviation, environmental protection, public health, cultural education, scientific research, science and technology extension, social development in rural and urban areas, and community construction. (1) With this continuous development and perfection of their social function, the public services in some NPOs present an increasing professionalisation. Consequently, some NPOs, taking public service as a core function, carry out various baseline public-benefit projects. This kind of NPO is exclusively classified as the first type: public welfare providing financial aid. (2) In addition, there are social groups and private non-enterprise units playing social functions in science and technology, education, environmental protection, culture and sports, medical treatment, social welfare, etc., as well as basic organisations offering a variety of community services. All these are defined as being of the second type: providing social services, for instance, hospitals, schools and training centres, parts of those centres providing social services, and health institutions in communities. (3) In the period of social transformation, NPOs, in various forms, have played increasingly extensive and direct roles in expressing wills, defending interests, coordinating relationships, resolving contradictions, and realising value in citizens. This is one of the important reasons for the rapid expansion in the number of the NPOs in China during recent years. With the development of this social function, promoting social coordination and participating in social governance are the main functions of some NPOs. Generally, there are two different types including community-based horizontal coordination and social group-based longitudinal ones in terms of their mechanism of action. The former involves all kinds of social organisations in communities, etc., and the latter includes various chamber of commerce, trade associations, trade unions, social gathering, student associations, and consumer associations. Such organisations are classed as NPOs which coordinate and manage society.

The first two types of NPOs, namely public welfare offering financial aid and NPOs providing social services are present in large numbers and are widespread. Their common features are a devotion to public service provision and a desire for public welfares to meet the needs of their beneficiaries. They beneficially supplement the public service of the government to a large extent by complementing government public services at all levels and across all relevant areas. The type of NPOs coordinating and managing society 
generally takes the association form of membership and focuses on social capital. They form a certain space of common interests and provide services based on the common interests of their members. Meanwhile, these NPOs, characterised by their large number, complexity, and strong self-stability, emphasise their representativeness in the communities or social groups where they actively participate in public affairs.

\subsection{Development Status}

2.2.1. Total Number: By the end of 2012, there were 13,683 NPOs in Suzhou, of which, 4,332 were registered NPOs and 9,351 were recorded community organisations, with 6.75 NPOs (registered) per 10,000 people (household registration). By the end of 2013, 5,825 NPOs were registered in Suzhou, of which, there were 2,721 social groups, and 3,104 private non-enterprise units. Suzhou had a total population of $6,478,100$; therefore, the number of NPOs (registered) per 10,000 people (household registration) reached 8.99. In addition, along with the 11,938 recorded community NPOs, there were 17,763 NPOs by the end of 2013. Compared with 2012, the total number of NPOs and the number of NPOs (registered) per 10,000 people (household registration) increased by $34.5 \%$ and $33.2 \%$, respectively [1]. Additionally, there were more than 400 foundations of various kinds registered with the Provincial Civil Administration Office of Jiangsu Province, China. In which, 72 foundations were in Suzhou, which approximately accounted for one sixth of those in the whole province.

2.2.2. Areas of Involvement: There were 72 foundations in Suzhou registered with the Administration Bureau of NPOs in Jiangsu Province. A total of 930 associations and private non-enterprise units can be found by searching on the Suzhou Civil Affairs website, of which, there were 500 associations and 430 private non-enterprise units [2]. Various types of NPOs have played an important role in all fields involving politics, the economy, society, culture, environmental protection, sports, etc., in Suzhou.

The data collected from the Administration Bureau of NPOs in Jiangsu Province in 2014 showed that there were 72 foundations registered in Jiangsu. Excluding nine foundations which were newly established and returned insufficient information, there were $30(47.6 \%)$ public offering, and $33(52.4 \%)$ non-public offering, foundations, respectively, within the total 63 foundations in Suzhou. Furthermore, there were 60 (95.2\%) foundations with local service scope and merely three $(4.8 \%)$ offering services nationally. However, these foundations are involved in diverse areas such as education, scientific research, medical aid, rural problems (village life and farming), public services, poverty alleviation, security and disaster relief, community development, culture, sports, arts, and so on. Meanwhile, the beneficiaries include the elderly, women, youth groups, children, the disabled, etc. [3].

The fields involved and the achievements achieved by the activities of the NPOs were summarised and analysed according to the Statistical Yearbook of Suzhou [4], as specified in Tables 1 to 4.

Field 1: education

Table 1. General Situation of Education in Suzhou during 2012-2013

\begin{tabular}{|l|l|l|}
\hline & 2012 & 2013 \\
\hline The number of various schools & 632 & 639 \\
\hline Colleges and universities & 20 & 21 \\
\hline Secondary vocational education schools & 33 & 30 \\
\hline Technical schools & 11 & 10 \\
\hline General middle schools & 261 & 261 \\
\hline Primary schools & 294 & 304 \\
\hline
\end{tabular}




\begin{tabular}{|l|l|l|}
\hline Special education schools & 13 & 13 \\
\hline School students & 989,146 & $1,026,996$ \\
\hline Colleges and Universities & 192,206 & 201,926 \\
\hline Secondary vocational education schools & 64,706 & 57,936 \\
\hline Technical schools & 27,244 & 25,069 \\
\hline General middle schools & 261,788 & 266,943 \\
\hline Primary schools & 440,400 & 47,2418 \\
\hline Special education schools & 2,802 & 2,704 \\
\hline Graduates & 224,189 & 226,286 \\
\hline Kindergartens & 465 & 509 \\
\hline Kindergarten children & 229,746 & 248,006 \\
\hline Artistic performance organisation & 14 & 14 \\
\hline Public library & 12 & 11 \\
\hline Museums & 36 & 40 \\
\hline
\end{tabular}

Field 2: medical and health services

Table 2. General Situation of the Medical and Health Services

\begin{tabular}{|l|l|l|}
\hline & 2012 & 2013 \\
\hline Health institutions & 2,992 & 3,001 \\
\hline Beds available in health institutions & 46,070 & 51,663 \\
\hline Health professionals & 57,186 & 61,051 \\
\hline Doctors & 23,194 & 27,296 \\
\hline Registered nurses & 21,943 & 24,097 \\
\hline Calculation according to resident population: & & \\
\hline Beds in health institutions per 1,000 people & 7.71 & 4.88 \\
\hline Hospital beds per 1,000 people & 6.01 & 4.28 \\
\hline Health professionals per 1,000 people & 8.82 & 5.77 \\
\hline Doctors per 1,000 people & 3.58 & 2.30 \\
\hline Registered nurses per 1,000 people & 3.39 & 2.28 \\
\hline
\end{tabular}

Field 3: social welfare

Table 3. General Situation of the Civil Affairs

\begin{tabular}{|l|l|l|}
\hline & 2012 & 2013 \\
\hline Social welfare institutions & 7 & 7 \\
\hline Social welfare hospitals & 1 & 1 \\
\hline Endowment institutions & 205 & 213 \\
\hline Beds of social welfare institutions & 3,255 & 4,281 \\
\hline Beds of endowment institutions & 46,223 & 51,840 \\
\hline Law firms & 194 & 206 \\
\hline Full time lawyers & 2,292 & 2,495 \\
\hline The number of People's Mediation committees & 5,379 & 8,374 \\
\hline
\end{tabular}

Field 4: community service 
Table 4. Situation of Social Service Centres

\begin{tabular}{|c|c|c|c|c|}
\hline Areas & Units (number) & $\begin{array}{c}\text { Number of } \\
\text { employees } \\
\text { (people) }\end{array}$ & $\begin{array}{c}\text { Income } \\
(¥ 10,000)\end{array}$ & $\begin{array}{c}\text { Other service facilities } \\
\text { of communities } \\
\text { (number) }\end{array}$ \\
\hline The whole city & 100 & 947 & 5,155 & 2,682 \\
\hline Urban area & 62 & 701 & 2,616 & 1,890 \\
\hline Wujiang area & 9 & 231 & 1,450 & 975 \\
\hline Changshu & 13 & 82 & 369 & 333 \\
\hline Zhangjiagang & 8 & 64 & 860 & 66 \\
\hline Kunshan & 10 & 39 & 1,050 & 80 \\
\hline Taicang & 7 & 61 & 260 & 313 \\
\hline
\end{tabular}

\subsubsection{Evaluation}

\section{(1) Annual Inspection System}

The NPOs of Suzhou are required to prepare material for annual inspection according to the Annual Inspection Instructions for Municipal Social Groups and Annual Inspection Instructions for Municipal Private Non-enterprise Units and to participate in the annual inspection/audit timeously. The results of the annual inspection of NPOs are divided into qualified, basically qualified, and fail, and are announced publically. Those NPOs behaving illegally need to be rectified timeously by the registration authority in accordance with prevailing law, while those allowing such deadlines to lapse are designated as failing at annual inspection. The registration authority carries out administrative punishment by deregistering those NPOs which do not participate in, or fail, the annual inspection for two consecutive years. Those basically qualified and failed NPOs in the annual inspection are not allowed to undertake the transfer of government functions or to buy public services from the government by bidding in the following year. The Municipal Bureau of Civil Affairs in Suzhou announced the results of the annual inspection of its municipal NPOs in 2012. The results indicated that there were 1,009 NPOs required to participate in the annual inspection, within which, 941 attended (a participation rate of $93.2 \%$ ). The results also showed that there were 937 qualified NPOs, accounting for $99.5 \%$ of the total participating; while there were four failed NPOs accounting for $0.5 \%$ of the total. The NPOs need to be rectified in those cases failing annual inspection for a single: they are deregistered when failing for two consecutive years.

\section{(2) Grade Evaluation System}

To enhance the capacity, and promote the healthy development, of NPOs, Management Methods for the Grade Evaluation of NPOs and Management Methods of Evaluating the NPOs in Jiangsu Province proposed by the State Civil Administration were adopted. The construction and evaluation of the NPOs of Suzhou were also considered. On this basis, the local government performed grade evaluation of the NPOs. The following criteria were used: The Implementation Measures for Evaluating the NPOs in Suzhou (revised in 2012), Detailed Regulations for Evaluating Industrial Associations in Suzhou (revised in 2012), Detailed Regulations for Evaluating Academic Associations in Suzhou (revised in 2012), Detailed Regulations for Evaluating Professional Associations in Suzhou (revised in 2012), Detailed Regulations for Evaluating Combined Associations in Suzhou (revised in 2012), Detailed Regulations for Evaluating Private Non-Enterprise Units in Suzhou (revised in 2013), Detailed Regulations for Evaluating the Chamber of Commerce in different areas of Suzhou, and Detailed Regulations for Evaluating Sports Associations in Suzhou (revised in 2012). The evaluation council of the NPOs of Suzhou evaluated the declaration units through self-evaluation and by declaration of the NPOs, field 
assessments from two levels of expert groups, and the rechecking of the expert groups dispatched by the Civil Affairs Bureau in 2012. The following conclusions were drawn: there were $65 \mathrm{~A}$-class NPOs, 48 4A-class ones (including 37 social groups and 11 private non-enterprise units), and 50 3A-class ones (including 35 social groups and 15 private non-enterprise units) [5].

\section{Development Problems Facing NPOs in Suzhou}

In recent years, the NPOs of Suzhou have flourished and their quantity almost doubled during the three years studied. However, they still faced many problems such as different concepts, insufficient quantity, scarce resources, weak capacity, inadequate supervision mechanisms, policy weaknesses, and so forth.

\subsection{Differences in Concepts and Understanding}

NPOs, as a new-type of entity in recent years, although developing rapidly, have failed to draw extensive attention of citizens and therefore exert an insignificant social influence. Some people still distrust NPOs in a traditional planned economic system and are wary of non-governmental organisations, fearing that NPOs upset the established order. Most people still uphold traditional ideas that some mass organisations and trade associations are the clubs of idlers and profit institutions in disguised forms and so on. The misunderstanding of ideas impedes the development of NPOs to some extent. In leadership, a minority of people pays inadequate attention to the position and effect of NPOs and this formulaic thinking remains embedded (in part) in government omnipotence. They are accustomed to depending on the government; meanwhile, they do not pay enough attention to the potential of NPOs: therein also lies a lack of trust.

\subsection{Too Few NPOs}

By the end of 2013, a total of 5,825 NPOs were registered with the civil affairs department in Suzhou, of which, there were 2,721 social groups and 3,104 private non-enterprise units. The number of NPOs (registered) per 10,000 people (household registration) reached 8.99 considering the total population of $6,478,100$ in Suzhou. Although rapid development has occurred in recent years, the number of NPOs per 10,000 people in Suzhou is still too small even compared with the data collected from developed countries and other regions 15 years ago (i.e. in 1999). It still presents obvious gaps compared to the 110, 97, 52, 25, 24, 14.5, and 13 NPOs per 10,000 people in France, Japan, United States, Argentina, Hong Kong, Singapore, and Brazil, respectively (see Table 5).

\section{Table 5. The Number of NPOs per 10,000 People in 15 Countries and Regions}

\begin{tabular}{|l|l|l|l|l|}
\hline $\begin{array}{l}\text { Countries and } \\
\text { regions }\end{array}$ & $\begin{array}{l}\text { Number of } \\
\text { NPOs }\end{array}$ & $\begin{array}{l}\text { The number of } \\
\text { people } \\
\mathbf{( 1 0 , 0 0 0 s})\end{array}$ & $\begin{array}{l}\text { The number } \\
\text { of NPOs per } \\
\mathbf{1 0 , 0 0 0} \text { people }\end{array}$ & Year \\
\hline France & $600000-700000$ & 5885 & 110.45 & 1999 \\
\hline Japan & 1228344 & 12641 & 97.17 & 1999 \\
\hline Belgium & 82000 & 1020 & 80.39 & 1999 \\
\hline United States & 1400000 & 27030 & 51.79 & 1999 \\
\hline Hungary & 35915 & 1011 & 35.52 & 1999 \\
\hline Germany & $180000-250000$ & 8205 & 26.20 & 1999 \\
\hline Indonesia & 350000 & 20368 & 17.18 & 1999 \\
\hline Singapore & 4600 & 316 & 14.56 & 1999 \\
\hline
\end{tabular}




\begin{tabular}{|l|l|l|l|l|}
\hline Brazil & 210000 & 16581 & 12.66 & 1999 \\
\hline India & 1000000 & 97967 & 10.21 & 1999 \\
\hline Taiwan, China & 20473 & 2192.9 & 9.34 & 1999 \\
\hline Suzhou, China & $\mathbf{5 8 2 5}$ & $\mathbf{6 4 7 . 8}$ & $\mathbf{8 . 9 9}$ & $\mathbf{2 0 1 3}$ \\
\hline Poland & 29580 & 3867 & 7.72 & 1999 \\
\hline Romania & 12000 & 2250 & 5.33 & 1999 \\
\hline Egypt & 18318 & 6140 & 2.44 & 1999 \\
\hline
\end{tabular}

\subsection{Scarce Organizational Resources}

The investigation of, and statistics from, 2008 showed that in Suzhou's municipal trade associations, those having preferable performances showing an annual income of $¥ 30,000$ to $¥ 50,000$, which were barely enough for daily expenses, while, one third of organisations cannot operate regularly [6]. To date, the interviews and investigations performed by the author revealed the difficulties facing NPOs in Suzhou in terms of financing and recruitment of personnel. The main financial sources of the majority of the organisations remained financial aid and appropriation provided by the government in addition to a small sum from social donations and membership fees. This serious lack of capital leads to difficulty in the normal running of NPOs and more difficulty when trying to sustain larger public projects. As a result, many organisations are dormant. Considering human resources, the staffs of most NPOs hail from government organisations; while grass-root NPOs are largely dependent on volunteers to develop their activities.

\subsection{Weakness in Capabilities}

Currently, China is in a stage of social transformation, during which, some NPOs fall foul of changing regulations or fail to implement their management and governance systems properly. Although some NPOs formulate regulations, they cannot implement them independently and their functions such as organisation, governance, coordination, and supervision fall far below the requirements of a modern market economy. In addition, a small number of NPOs have non-public internal management, imperfect financial systems, and low transparency. The staff of NPOs are generally lacking in innovative ideas and knowledge, are used to the status quo: there is a distinct lack of stomach for reform. Meanwhile, they clearly know their own shortcomings, which urgently call for relevant training including the latest developments from NPOs abroad, their experience in operating projects, and the design and management of their organisation systems. Furthermore, some NPOs have an aging staff profile, low professional quality, small membership coverage, etc. In short, the human resource situation of NPOs lags far behind the development requirements of organisations in today's society: adapt to a new environment is this difficult.

\subsection{Lack of Evaluation and Supervision Mechanisms}

Unlike other enterprises, the performance of NPOs cannot usually be measured through profits owing to the public welfare role. Therefore, to measure their performance, an effective evaluation system needs to be established. Currently, Suzhou mainly uses the annual inspection and grade evaluation systems to assess the NPOs. However, the civil administration department failed to review the reports of annual inspections individually due to the large number of organisations; instead, the annual inspection became routine. In addition, the single evaluation system has a lack of multi-dimensional evaluation systems such as those involving third-party and peer discipline. Furthermore, there is no effective social supervision. Once an NPO is approved and established, relevant departments seldom monitor and manage 
the daily business operations thereof. On the one hand, there is no independent third-party supervisory institution in China; on the other hand, the public has not yet formed strong supervisory awareness, and the media, as the extension of central administrative functions, fails to report issues around NPOs.

\subsection{Insufficient Policy Support}

The 18th National Congress of the Communist Party of China proposed a policy accelerating the formation of a modern NPO system with separated government and community roles, clear responsibilities, and legal autonomy. To implement this in spirit, and to give full reign to the positive effect of NPOs, strengthen the social construct, and create social management structures, the Suzhou Municipal Government has also published a raft of policies to guide and support the development of NPOs in recent years. These include: Advice on Further Strengthening the Social Construct and Creating Social Management of NPOs (published by Suzhou [2011] No. 30), Advice on Further Strengthening the Construction of NPOs in Suzhou (published by the Municipal Office of Suzhou [2011] No. 63), Several Advice Notes on Accelerating the Healthy Development of NPOs in Suzhou (published by the Municipal Office of Suzhou [2013] No. 81), Guiding Opinions on Vigorously Developing NPOs (published by the civil administration of Suzhou [2012] No. 251), etc. In addition, all areas also publish supporting measures, such as: Supporting Measures for NPOs in Suzhou Industrial Zones, Implementation Measures for Vigorously Developing NPOs in Wujiang Area, Supporting Policy for the Development of the NPOs in Gusu area (Trial), and the Implementation Details of the Talent Plan for NPOs in the Gusu area of Suzhou (Trial). These supporting measures and policies have been unveiled in the past three years. Although they were published quickly, they remain insufficient for the rapid development of NPOs. Firstly, many new policies are in trial implementation period; therefore, it remains uncertain as to whether they are suitable for the development of NPOs, and their subsequent effects need to be evaluated. Secondly, the government offers less support, an unbalanced allocation of social resources, and inadequate financial support for NPOs compared with other types of enterprises.

\section{Countermeasures and Suggestions for the Development of NPOs in Suzhou}

\subsection{Enhancing Public Opinion: the NPO Perspective}

A focus on the combination of social public undertakings and enterprise culture and development of multi-domain, diversified public-benefit projects by fully utilising and integrating social resources such as the media, the public, volunteers, and so on, is suggested. On the basis of improving the degree of participation of citizens, it is expected to expand the influence of each organisation by enhancing the operational level of projects and strengthening organisational reputation-building [7]. Therefore, it is necessary to update concepts, seize opportunities, and intensify propaganda so as to further enhance public understanding of NPOs. A favourable external environment is expected to be created by obtaining public supports based on their acceptance and approval of NPOs. Moreover, publicity can be enhanced through media, and selfreporting of NPOs. Furthermore, further promotion of the interaction of the trade associations in the Yangtze River Delta area is promoted by actively engendering their cooperation and using exchanges to enhance their influence. Additionally, a strengthening of the cultivation of NPOs by community network-building is proposed. 


\subsection{Capacity Building from Within}

It is also extremely important to strengthen the self-development of NPOs, which have developed rapidly in Suzhou since the onset of economic reform and openness. NPOs are required to strengthen organisation-building and improve their overall quality so as to develop healthily. Such organisations are asked to formulate their detailed regulations in advance of performing capacity building and revise them regularly. They are also asked to perfect the contents of such regulations and policies, and in particular review periodical goals and service levels delivered so as to enhance their own senses of responsibility and self-discipline. The core staff in these organisations need to carry out activities in strict accordance with established rules, and to follow their professional ethical codes, follow organisational discipline, abide by the regulations of management, and uphold a code of conduct. The employees at all levels are expected to fulfil their responsibilities according to the goals established by organisational regulations. On this basis, the NPOs are required to plan, implement, and summarise the tasks at each stage and to discuss and correct problems as they emerge in a flexible, proactive fashion. By doing so, the staff can continuously learn, exchange ideas, and share at work, while constantly gaining experience. Meanwhile, regular internal training and team-building, and the encouragement of employees to participate in external capacity-building activities are suggested. In this way, it is expected to enhance cohesion within such organisations by providing a platform for the growth in staff numbers in addition to promoting their abilities.

\subsection{Constructing Systems for Introducing and Training Personnel}

The enhanced independence of NPOs calls for a gradually independent powers of appointment and removal of personnel. In other words, the organisations must introduce appropriate personnel according to their own developmental and operational needs without the intervention of the government, funders, or donors. Based on independent staff appointment and removal, the governance and management layers of NPOs need to accommodate the establishment of human resources type appointments. Moreover, they are required to attract professional or appropriate personnel and those who identify with organisational goals by a variety of recruitment channels, and to be good at playing volunteer roles. Currently, the staff of NPOs in China can participate in training in three ways: long-term formal education, short-term training, and authentication training. For NPO employees, taking part in short-term courses for capacity-building is still the most common training method. These courses are aimed at the problems of management, technology, development, etc. Most audiences impose low-level requirements, and most courses are either free or cheap. However, various agencies offer uneven course qualities due to their different qualifications and because their training systems need to be improved and updated. Therefore, enriching and perfecting training systems for capacitybuilding, especially for short-term training courses and training certification, are conducive to broadening modes of learning among employees. In this way, training is expected to play a direct role in promoting NPO capacity-building.

\subsection{Establishing and Perfecting Evaluation and Supervision Mechanisms}

Establishing effective evaluation and supervision mechanisms is critical for NPOs to obtain public trust and respect. It is suggested that NPOs establish supervision and management teams by taking the key players from government as their group leaders and drawing on senior staff in departments such as organisation, discipline, inspection and supervision, civil administration, finance, taxation, auditing, public security, national security, and so on as their main members. In order to ensure efficacious supervision and management of NPOs, it is necessary to intensify communication and coordination [8], clarify respective tasks and responsibilities, and explore the establishment of affiliated 
cooperative mechanisms. Meanwhile, overseas experience shows that it is effective to supervise and evaluate NPOs using the power of the public. Government departments can request that NPOs publicise relevant information and establish channels for public complaint through telephone or the Internet. In addition, specific posts are set-up to deal with complaints and publish them, and the outcome, on the Internet. In this way, an institutionalised, operable evaluation system is expected to emerge. Meanwhile, it is necessary to give full play to the supervision function of the media and further intensify their potential to publicise the work of NPOs. They are required to not only report the touching deeds of those NPOs but also disclose, in a timely manner, the illegal behaviour of some others.

\section{Conclusions}

With the establishment of a socialist market-economic mechanism, the political system reforms have transformed government functions. It is impossible, and indeed inappropriate, for government to manage all social affairs directly, and thus NPOs can undertake a large number of public management functions. This phenomenon calls for changes in the model of social management by a government used to a monopoly of power in the past. It is necessary to gradually construct a good governance model with reasonable divisions of responsibility and co-governance between the government and other main players through reform and innovation. By doing so, a new pattern of social governance with economic marketisation, political democratisation, and societal selfgovernment is expected to be formed through gradual interaction among multiple subjects involving government, enterprises, and NPOs. At present, active guidance and development of NPOs are needed by the society they serve. To promote a more standard, orderly, and healthy development path for NPOs in Suzhou, it is suggested that the government successively unveil relevant supporting policies in the future.

\section{Acknowledgments}

This work was supported in part by a grant from National Natural Science Foundation of China (Number: 71002017). This work was supported in part by a grant from Philosophical and Social Science Foundation of University in Jiangsu Province (Number: 2014SJB548). This work was supported in part by a grant from "333" Projects Foundation of Jiangsu Province. (Number: BRA2015417).

\section{References}

[1] A. Rikki, "The nonprofit sector and the informal Sector: a theoretical perspective", Voluntas, vol. 10, (1999), pp. 131-148.

[2] C .Claxton and J. Feder, "Public policy issues in nonprofits conversions: an overview", Health Affairs, vol. 16, (1997), pp. 9-28.

[3] D. Hui, "Study on the development of trade associations of Suzhou city", Southeast Commuication, vol. 5, (2007), pp. 145-146.

[4] J. Ferris and E. Graddy, "The role of the nonprofit sector in a selfgoverning society: a view from the United States", Voluntas, vol. 9, (1999), pp. 137-154.

[5] H. Weizhong, "The research on relationship between philanthropy organization and the government", Economy and Society Development, vol. 4, (2009), pp. 113-115.

[6] J. Xijin, "The third development path of China civil society", Chinese Public Administration, vol. 3, (2003), pp. 22-23.

[7] W. Zhihui, "Suzhou's trade associations: problems and recommendations", China Nonprofit Review, vol. 2, (2009), pp. 188-203.

[8] Z. Fei and T. Xueying, "Development status and countermeasures of the non-profit organization in Suzhou", Economic Research Guide, vol. 9, (2014). 